\title{
Developing automatic recognition system of drill wear in standard laminated chipboard drilling process
}

\author{
J. KUREK ${ }^{1}$, M. KRUK ${ }^{1}$, S. OSOWSKI ${ }^{2 *}$, P. HOSER ${ }^{1}$, G. WIECZOREK ${ }^{1}$, A. JEGOROWA ${ }^{3}$, \\ J. GÓRSKI ${ }^{3}$, J. WILKOWSKI ${ }^{3}$, K. ŚMIETAŃSKA ${ }^{3}$ and J. KOSSAKOWSKA ${ }^{4}$ \\ ${ }^{1}$ Faculty of Applied Informatics and Mathematics, Warsaw University of Life Sciences, 166 Nowoursynowska St., 02-787 Warsaw, Poland \\ ${ }^{2}$ Faculty of Electrical Engineering, Warsaw University of Technology and Faculty of Electronic Engineering, Military University \\ of Technology, Warsaw, 75 Koszykowa St., 00-662 Warsaw, Poland \\ ${ }^{3}$ Faculty of Wood Technology, Warsaw University of Life Sciences, 166 Nowoursynowska St., 02-787 Warsaw, Poland \\ ${ }^{4}$ Institute of Manufacturing Technologies, Warsaw University of Technology, 85 Narbutta St., 02-524 Warsaw, Poland
}

\begin{abstract}
The paper presents an automatic approach to recognition of the drill condition in a standard laminated chipboard drilling process. The state of the drill is classified into two classes: "useful" (sharp enough) and "useless" (worn out). The case "useless" indicates symptoms of excessive drill wear, unsatisfactory from the point of view of furniture processing quality. On the other hand the "useful" state identifies tools which are still able to drill holes acceptable due to the required processing quality. The main problem in this task is to choose an appropriate set of diagnostic features (variables), based on which the recognition of drill state ("useful" versus "useless") can be made. The features have been generated based on 5 registered signals: feed force, cutting torque, noise, vibration and acoustic emission. Different statistical parameters describing these signals and also their Fourier and wavelet representations have been used for defining the features. Sequential feature selection is applied to detect the most class discriminative set of features. The final step of recognition is done by using three types of classifiers, including support vector machine, ensemble of decision trees and random forest. Six standard drills of $12 \mathrm{~mm}$ diameter with tungsten carbide tips were used in experiments. The results have confirmed good quality of the proposed diagnostic system.
\end{abstract}

Key words: diagnostic expert systems, neural networks, wavelet packets, wear monitoring.

\section{Introduction}

One method to achieve higher quality and productivity of machine tools is applying wear monitoring diagnostic systems, especially TCM (tool condition monitoring) and PCM (process condition monitoring). The aims of TCM include the identification of beginning and end of the cutting process, detection of catastrophic tool failure, quality control of cutting process, etc. - all realized at low cost $[1,2]$.

The dynamic development and the increasing use of flexible automation in wood production process lead to higher interest in automatic monitoring of cutting tools [3-5]. However, the complexity of production process and influence of different interferences make existing diagnostic systems not fully satisfactory. There is still need to conduct research directed for developing better diagnostic methods of tools used in wood industry, especially the drills.

Drill wear results in decreasing the cutting edge properties, being one of the most important issues in machining science $[3,6]$. The blunting is caused by mechanical, thermal and chemical influence of the work piece, especially when wood based materials are machined. Wide abrasion due to the existence of hard mineral contaminations in material, glue, and friction of wood is observed. Increasing content of glue and mineral contaminations in external layers of wood based

*e-mail: sto@iem.pw.edu.pl materials is a reason behind much more intensive edge wearing $[5,7]$.

The key issue in diagnostics of cutting tool state is the selection of sensors. Usually force sensors, electrical power, acoustic emission, vibration and acoustic pressure are applied in TCM $[4,8]$. The best results in metal working are achieved using force sensors.

The disadvantage of this kind of measurement in normal production is that the force or torque transducers are relatively expensive and there are difficulties in mounting these sensors to the cutting tool or work piece. Therefore vibration sensors - easy to install, but at the same time less accurate because of background noise - are most commonly used in wood industry [5]. Vibration measurement is easy, since an accelerometer can be mounted close to the spindle bearing and no modifications of machine tools are needed [9].

Quite similar to vibration is the sound signal, which can also be used in assessment of drill state. Mechanical vibration of the cutting tool, machine holder and drill are partly transferred to airborne vibration. It means that part of the information contained in vibration can also be obtained from sound measurement. The acquisition of the sound can be done easily using a microphone. However, the sound pressure sensors (microphones), are even more susceptible to background [7, 8]. The sound measurement in an extended range of frequencies from $20 \mathrm{kHz}$ to $80 \mathrm{kHz}$ is usually applied [4]. This range is called the acoustic emission. The effectiveness of acoustic emission sensors in wood industry is still controversial $[5,7]$. 
The signals of the sensors mentioned are correlated with the state of the tool, however, they are susceptible to random interferences and background noise. Therefore, many types of sensors are used in a parallel. In this way the information of the tool state is enhanced.

Based on sensor signals the diagnostic features are created and used as the input attributes to the final classification system. In many publications diagnostic features are defined directly on the basis of sensor signals in time domain. Statistical parameters such as mean value, root mean squared value, power, energy, kurtosis, skewness, etc., are exploited. In some applications different threshold levels for signal values are defined. Then the number of crossing these levels by the signal, as well as the time spent by signal in the areas between different levels, is used as the diagnostic features [4]. The features are also generated on the basis of Fourier transformation of the sensor signals. They are defined in the form of harmonic values, power spectral density, cepstrum or other statistical descriptions in several dominant bands [10]. High correlation of some spectral parameters with the state of the cutting edge of the drill was demonstrated $[1,2]$.

Currently wavelet and wavelet packet transformations are also used in defining the diagnostic features of the tools $[4,11]$. Wavelet transformation generates many signal bands, which are the basis for creating the statistical diagnostic features.

Application of different methods in feature generation enriches the information of the production process and also of the tool state. Using them as the input attributes to the final recognition (classification) units allows for building more sophisticated diagnostic systems. Different classifiers have been used in this application. The most popular is application of neural networks and fuzzy systems [8, 12-15] or autoregressive model [16].

In this research we apply extended types of diagnostic features defined based on five sensor signals registered in a laminated chipboard drilling process. The following physical quantities have been measured and used in generation of the diagnostic features:

- feed force (denoted as F),

- cutting torque (denoted as M),

- noise (denoted as C),

- vibration (denoted as V),

- acoustic emission (denoted as AE),

Many features can be generated automatically on the basis of these signals. However, some of them may represent no diagnostic information. Hence the selection of the most important features is an important step in building the efficient diagnostic system.

Good feature should differentiate classes of drill states. In this research we consider two classes: "useful" (class 1) and "useless" (class 2). The first class refers to a tool which is still sharp enough to drill holes which are acceptable from the point of view of processing quality. The second class denotes unaccepted drill state resulting in quality unsatisfactory from the point of view of the final product.

The paper will present the process of building an automatic diagnostic system, able to assessing the state of the drill with the satisfactory accuracy. It starts from acquisition of sensor signals, then describes the generation of diagnostic features, selection of best set of these features and finally recognition of the state of drill ("useful" versus "useless") based on the pattern represented by the selected set of features. Three different classifiers are checked and compared in the role of recognizing unit: the ensemble of ordinary decision trees, random forest of multivariate decision trees and support vector machine.

\section{Measurement methodology}

All data used in experiments have been acquired using standard Buselatto JET $100 \mathrm{CNC}$ vertical machining centre. The experiments have been performed on laminated chipboard using standard drills of $12 \mathrm{~mm}$ diameter with tungsten carbide tips ("FABA" - Poland). The chipboard and drill chosen are depicted in Fig. 1.

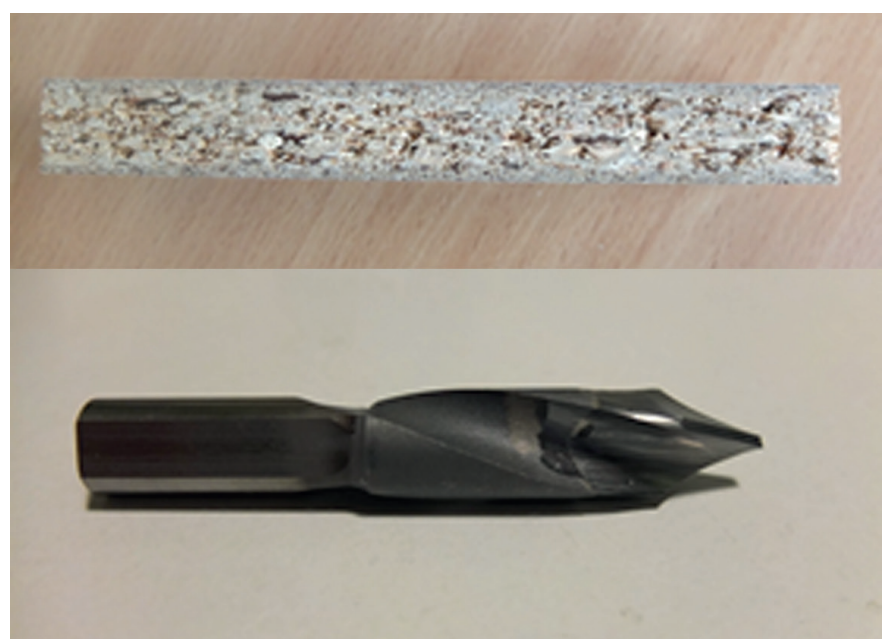

Fig. 1. Standard laminated chipboard and drill used in experiments

To measure all required physical quantities special sensors have been applied. The experimental set-up was composed of the following elements:

- AE-acoustic emission measuring system (Kistler 8152B contact sensor, Kistler 5125B amplifier),

- V-mechanical vibration measuring system (Kistler 8141A accelerometer, Kistler 5127B amplifier),

- C-noise (sound pressure) measuring system (B\&K 4189 microphone and preamplifier, B\&K NEXUS 2690 amplifier),

- F and M - dynamometer with Kistler 9345A sensor and ICAM5073A amplifier.

The acquisition of sensor signals was done using two data acquisition cards (NI PCI-6111 and NI PCI-6034E) through connection blocks (NI BNC-2110), as shown in Fig. 2.

The use of two acquisition cards was due to different sampling frequencies: $2 \mathrm{MHz}$ for acoustic emission signal and 50 $\mathrm{kHz}$ for vibration and noise signal. LabVIEW National Instruments application has been prepared to register all required signals. 

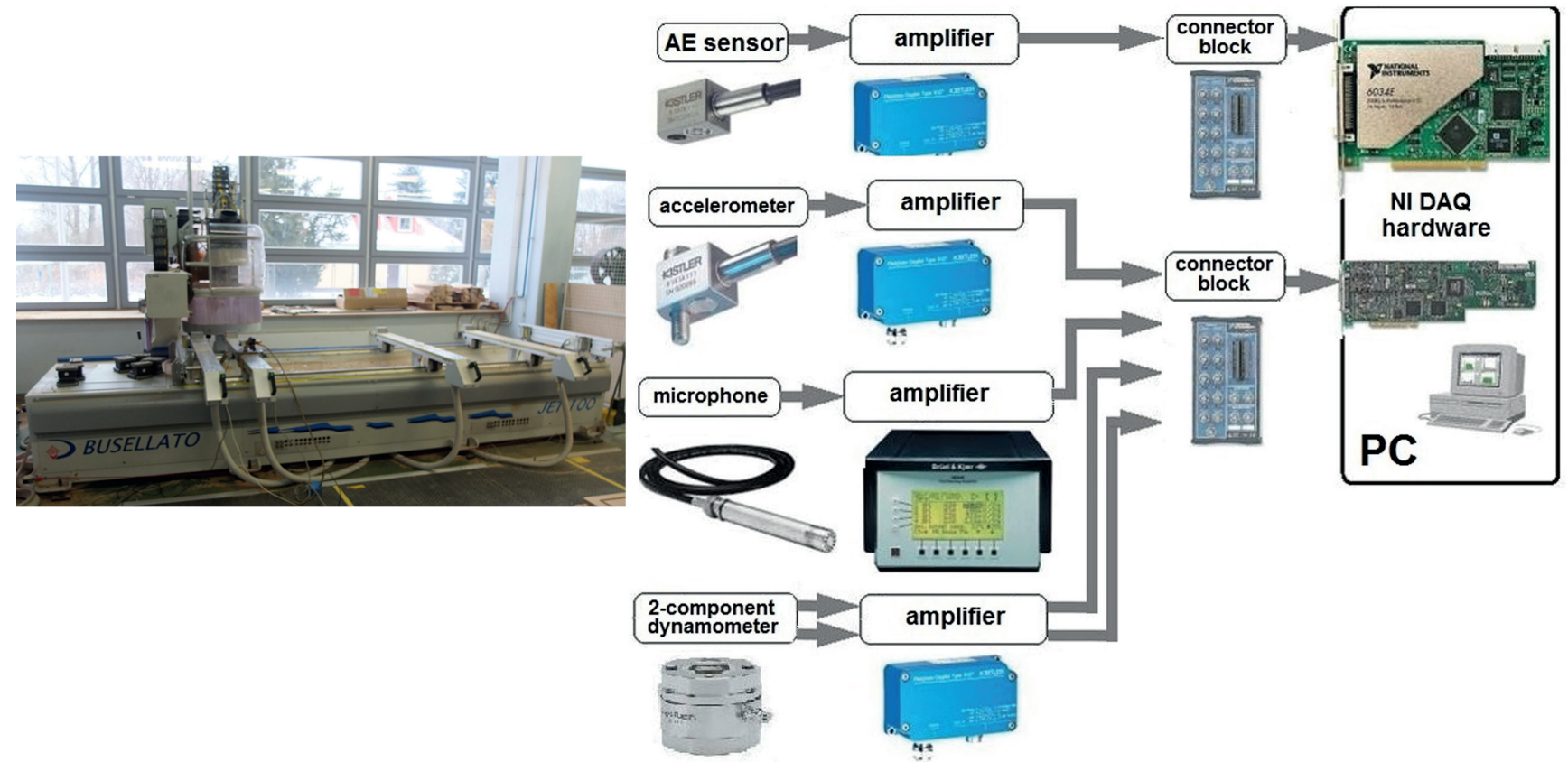

Fig. 2. The scheme of experimental set-up

The main goal of research is to create an automatic system, which is able to recognize two states of drill: "useful" (class 1) and "useless" (class 2). The quality of drilling results was controlled manually by a human expert taking into account the smoothness of the circumference and tolerance parameters specified in furniture manufacturing. Examples of acceptable and unacceptable holes drilled in a laminated chipboard are shown in Fig. 3.

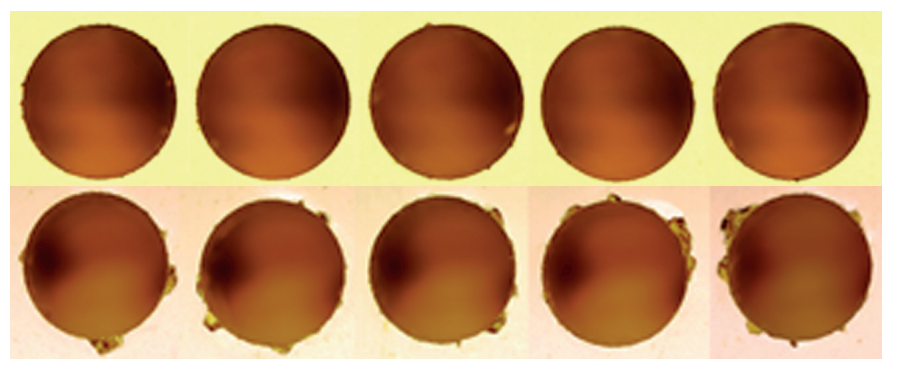

Fig. 3. Examples of acceptable (upper row) and unacceptable (lower row) holes drilled in a laminated chipboard

\section{Database for experiments}

The database for numerical experiments was prepared using 6 drills which were used repeatedly to generate the proper learning data used in further processing. In the first phase, all new drills were used in laminated chipboard drilling process and the proper sensor signals (feed force, cutting torque, noise, vibration and acoustic emission) have been registered. Five repetitions of this process have been registered. All these samples represented observations belonging to class one.
One drill (No 6) treated as the reference was left without blunting and five other (No 1-5) were subject to gradual blunting by means of more and more holes drilling. Drills have been blunted successively in a multiphase way. After each phase of blunting the drilling process was repeated 5 times and signals of five sensors were registered as the samples representing class 1 ("useful") or class 2 ("useless") according to the opinion of the human expert, who was responsible for dichotomous evaluation of processing quality (acceptable versus unacceptable). The reference tool (drill No 6) was used only for 27 holes drilling in order to avoid any significant symptoms of blunting. Hence, it was the only drill which was "useful" throughout the entire experimental study. The size of the registered time samples arranged in a vector forms was the same and equal to 1000 .

The performed trials created the database used in further numerical experiments. Due to different quality of drills the structure of the database was formed in the following way.

- Drills Nos 1, 2 and 5

5 trials at 3 stages of "useful" (sharp enough) drill state $(3 \times 5=15$ signal registrations belonging to class 1$)$ and 5 trials at 6 stages of "useless" (excessively worn) drill state $(5 \times 6=30$ signal registrations belonging to class 2$)$. Total number of measurements for 3 above-mentioned drills: $3 \times 45=135$.

- Drill No 3

5 trials at 4 stages of "useful" drill state $(5 \times 4=20$ signal registrations belonging to class 1$)$ and 5 trials at 4 stages of "useless" drill state $(5 \times 4=20$ signal registrations belonging to class 2 ). Total number of measurements for this drill: 40.

- Drill No 4

5 trials at 2 stages of "useful" drill state $(5 \times 2=10$ signal registrations belonging to class 1 ) and 5 trials at 7 stag- 
es of "useless" drill state $(5 \times 7=35$ signal registrations belonging to class 2 ). Total number of measurements for this drill: 45.

- Drill No 6 (reference drill)

27 signal registrations for "useful" drill state.

In this way $102(3 \times 15+20+10+27)$ measurements of five sensor signals referred to class 1 have been registered. On the other hand $145(3 \times 30+20+35)$ trials referred to class 2 were observed and analyzed. It is worth noting, that drill No 3 was destroyed earlier than other drills used in the experiments - it means less trials than for others.

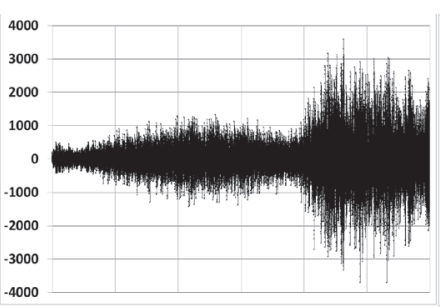

a)

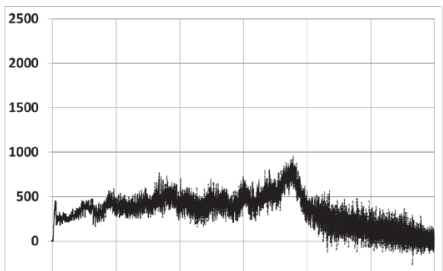

b)

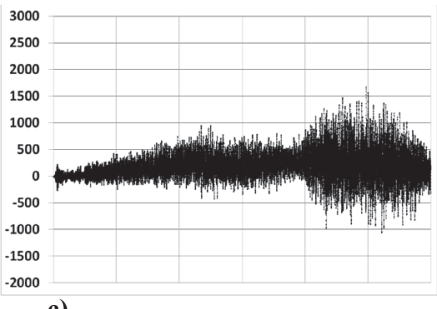

c)

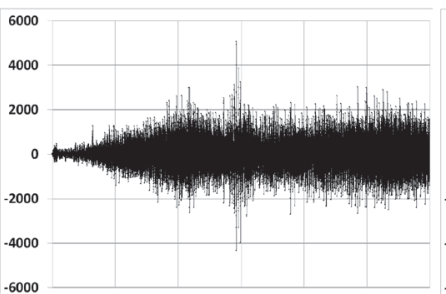

d)

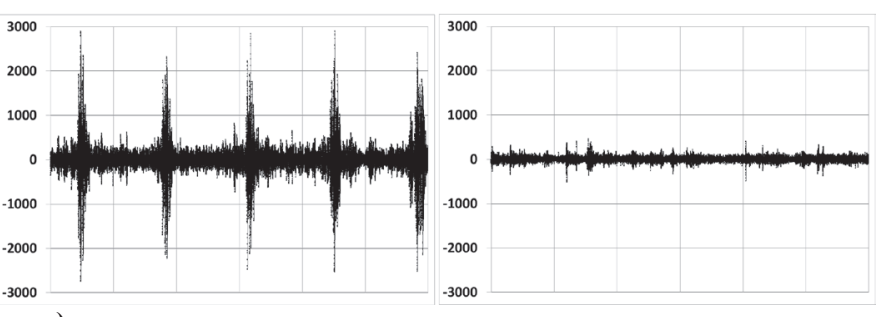

e)

Fig. 4. The signals of a) noise, b) feed force, c) cutting torque, d) vibration, and e) acoustic emission representing the sharp state of drill (left column) and worn out state (right column); horizontal axis represents time
Typical signals representing 5 physical quantities in absolutely sharp (factory fresh) and extremely worn state of drill are presented in Fig. 4. The first four signals have been registered at $50 \mathrm{kHz}$ sampling in the measuring window of $1.1 \mathrm{~s}$. The last signal representing acoustic emission was acquired at $2 \mathrm{MHz}$ sampling in a measuring window of $0.3 \mathrm{~s}$. All signals are expressed in millivolts.

\section{Feature generation}

The signals registered in all trials create the basis for generating the diagnostic features, which are used in an automatic recognition of drill state. Based on five physical quantities a lot of features can be generated. The first set of features was created directly on the basis of the time series of signals. The following features have been generated in this way (each representing five sensor signals):

1. Arithmetic mean of signals ( 5 features)

2. Standard deviation of signals ( 5 features)

3. Variance of signals ( 5 features)

4. Histogram (50 features)

5. Skewness of signals ( 5 features)

6. Kurtosis of signals ( 5 features)

7. RMS of signals ( 5 features)

8. Ratio of peak magnitude to RMS of signals (5 features). The histogram was created by splitting the data into 10 bins. The histogram features have been defined as the number of samples forming each bin. At 10 bins and 5 sensor signals 50 features have been generated in this way.

85 diagnostic features based on the time domain description have been created. The next set of features was generated on the basis of Fourier description of all five signals. Based on fast Fourier transformation (FFT) we have generated diagnostic features representing the number of frequencies in FFT spectrum, which exceeded 10 defined levels of threshold. They have been defined in the following way

$$
\begin{aligned}
F F T_{n} & =\operatorname{count}(f>\operatorname{mean}(\operatorname{abs}(F F T)+ \\
& +(n+2) \operatorname{std}(\operatorname{abs}(F F T))
\end{aligned}
$$

for $n=1,2, \ldots, 10$. The threshold values changed from 3 standard deviations (std) of spectrum, step one up to 13 standard deviations (10 different features for 5 sensor signals resulting in 50 additional features).

The next descriptors of the signals have been generated using the wavelet packet decomposition [17]. The advantage of wavelet transformation is analyzing the time series on many levels of time scale, which represent different ranges of time and frequency. Thanks to this it is possible to capture some hidden features contained in the signals in wider range of frequency.

Wavelet packets decompose the analyzed signal into the vectors of detail coefficients and approximation coefficients of coarser scale. In the subsequent steps the process of splitting is repeated on the approximation and detail vectors. Different families of discrete wavelet functions have been tried. They 
include Daubechies, symlets, coiflets, biorthogonal and Meyer functions of different orders $[17,18]$.

After decomposing signals into wavelet representation, the next features have been defined on the basis of energy of signals in all terminal nodes of the best level of decomposition. Second level wavelet packet decomposition was identified as the best for all applied wavelet functions. The experiments have been performed using Matlab [19]. In such case each $j$-th node $(j=1,2,3,4)$ on this level was characterized by the energy, according to the formula

$$
E_{j}=\sum_{k=1}^{N_{j}} S_{j k}^{2}
$$

where $S_{j k}$ is the coefficient of discrete wavelet packet transformation on the chosen (second) level, representing $j$ th node. $N_{j}$ is the length of coefficient vector in $j$ th node. It means that for one signal 4 values of energy are defined. In the case of 5 signals ( 5 sensors) 20 features have been generated in this way.

We have verified 18 various wavelet functions combined with time and frequency descriptors of the signals. Each set consisted of the same subset of variables generated on the basis of time and frequency representations and only the wavelet family was changed. In this way 18 different sets of potential diagnostic features have been generated. From these sets we have selected 7 sets which were most efficient in class recognition, depicted in Table 1.

Summarizing, 85 features were derived from time domain, 50 features generated from frequency domain and 20 features from wavelet representation. Overall, in this research we have investigated 155 descriptors, representing potential features used in class recognition.

The next step is to choose the optimal (reduced) set of features, selected from the mentioned above, which can separate two classes of drill state: sharp and worn out with the highest accuracy. From the set of 155 potential features we had to choose the best subsets characterizing classes in the most distinctive way.

\section{Feature selection}

To choose the best class discriminative set of features we have applied sequential feature selection. This approach detects a subset of features that predicts the classes by selecting sequentially features until there is no further improvement in class prediction accuracy [19]. Any solution of the classifier may be applied in this prediction process. Starting from an empty feature set, the feature selection creates candidate feature subsets by sequentially adding and removing each of the features not yet chosen. Each candidate feature subset is checked in 10-fold cross-validation by repeating the prediction process with different training and testing subsets of observations.

In general two types of operations are made within this process:

- forward selection, starting with no variables in the model, testing the significance of addition of each variable, adding the variable which improves model the most, and repeating this process until none variable improves the built model according to the assumed criterion.

- backward elimination, starting with some candidate set of variables, testing the deletion of each variable by using a chosen criterion of model quality, deleting the variable which improves the model to the highest extend by being deleted, and repeating this process until no further improvement is possible.

Both operations interlace each other. In each stage of the process, after a new variable is added, a test is made to check if some variables from the actual set can be deleted without increasing the error of regression. The procedure terminates when the quality measure of the classification model is maximized, or when the actual improvement is below some assumed tolerance value.

The entering and removing the particular variable from the actual feature set is controlled by two parameters: penter and premove [19]. The penter specifies the maximum $p$-value for a variable to be recommended for adding to the model. The premove specifies the minimum $p$-value for a variable to be

Table 1

The feature selection results based on time, frequency and wavelet representations of signals

\begin{tabular}{|c|c|c|}
\hline No & Wavelet & Features \\
\hline 1 & db1 & 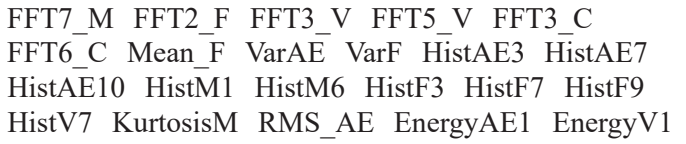 \\
\hline 2 & db5 & $\begin{array}{l}\text { FFT4_AE FFT7_M FFT2_F } \\
\text { FFT3_F }\end{array}$ \\
\hline 3 & $\mathrm{db} 20$ & $\begin{array}{l}\text { FFT4_AE FFT4_M FFT5_M FFT7_M FFT2_F } \\
\text { FFT4_F FFT5_F FFT3_V FFT4_V FFT1_C FFT3_C } \\
\text { FFT6_C Mean_F VarAE VarF HistAE6 HistAE7 } \\
\text { HistAE10 HistF3 HistF7 HistF8 HistF9 HistV7 } \\
\text { HistV8 KurtosisM KurtosisC RMS_AE EnergyAE1 } \\
\text { EnergyV1 }\end{array}$ \\
\hline 4 & coif4 & $\begin{array}{l}\text { FFT4_AE FFT7_M FFT2_F FFT3_V FFT4_V } \\
\text { FFT10_C Mean_F VarF VarC HistAE7 HistF3 } \\
\text { HistF7 HistF9 HistV7 KurtosisM RMS_AE } \\
\text { RMS_C EnergyV1 }\end{array}$ \\
\hline 5 & sym2 & $\begin{array}{l}\text { FFT4_AE FFT7_M FFT2_F } \text { FFT3_V FFT4_V } \\
\text { FFT10_C Mean_F VarF VarC HistAE7 HistF3 } \\
\text { HistF7 HistF9 HistV7 KurtosisM RMS_AE } \\
\text { RMS_C EnergyV1 }\end{array}$ \\
\hline 6 & $\begin{array}{l}\text { discrete } \\
\text { Meyer }\end{array}$ & $\begin{array}{l}\text { FFT4_AE FFT7_M FFT2_F } \text { FFT3_V FFT5_V } \\
\text { FFT10_C Mean_F VarF VarC HistAE7 HistF2 } \\
\text { HistF3 HistF9 HistV7 KurtosisM RMS_AE } \\
\text { RMS_C EnergyV1 }\end{array}$ \\
\hline 7 & rbio1.3 & $\begin{array}{l}\text { FFT2_F FFT3_V FFT5_V FFT3_C FFT6_C } \\
\text { Mean_M Mean_F VarAE VarF HistAE6 HistAE10 } \\
\text { HistM1 HistM10 HistF3 HistF7 HistF9 HistV7 } \\
\text { RMS_AE peak2RMS_M EnergyAE1 EnergyV1 }\end{array}$ \\
\hline
\end{tabular}


removed from the set. The result of the method is a logical vector indicating the finally chosen features.

Sequential feature selection was applied for different sets of features created from statistical time domain descriptors and Fourier descriptors, both combined with selected family of wavelets. Different wavelet functions have been checked. The best results of feature selection for different combinations of wavelet families are presented in Table 1. The first symbols in these notations refer either to the time domain (Mean, Var, RMS, Kurtosis, Skewness, Hist, peak2RMS), Fourier descriptors (FFT followed by the number of threshold level $n$ ) or wavelet descriptors (prefix Energy followed by suffix denoting the node number on second level of wavelet packet decomposition) of signals. The next symbols refer to the sensor signal (AE, F, V, M, and C). For example FFT4_AE means Fourier descriptor of AE signal associated with $4^{\text {th }}$ level of threshold $(n=4)$. The selected feature EnergyV1 refers to wavelet descriptor of signal $\mathrm{V}$ associated with $1^{\text {st }}$ node on the second level of wavelet packet decomposition at application of wavelet function shown in the second column of Table 1.

As we can see all sensor signals and all methods of descriptor creation take part in the selected sets. Relatively high participation is related to FFT representation and histogram of the signals. The particular contents of selected features depends on the actually used wavelet function applied in wavelet decomposition (the second column of Table 1).

\section{Classifiers}

To obtain the best possible results of class recognition we have tried different classification systems. Three different solutions have been compared: support vector machine (SVM), ensemble of classical decision tree (DT) and random forest of multivariate decision trees (RF). Nowadays all of them belong to the best classification systems [22].

6.1 Support vector machine. The assignment of every drill trial to one of two sharp/worn out classes has been done by applying the support vector machine of the Gaussian kernel [20-22]. SVM is a simple circuit structure of one hidden kernel layer and one output unit performing the weighted summation followed by sign function (positive summed signal means class 1 and negative - class 2). The hyperparameters (the regularization constant $\mathrm{C}$ and Gaussian kernel width) have been adjusted by repeating the learning experiments for the set of their predefined values and choosing the best one for the validation of data set.

The learning process of SVM network is relatively easy and effective since the whole learning task is simplified to the solution of the quadratic optimization problem with linear constraints. In our experiments we have used the modified Platt algorithm, implementing the modified sequential optimization [21].

6.2 Ensemble of decision tree. A decision tree is a classification tool that uses a tree-like graph for making decisions [23]. The leaves represent class labels and branches represent conjunctions of features that lead to these class labels. A tree is learned by splitting the source set into two subsets based on an attribute value test according to the adjusted threshold value. The process is repeated on each derived subset in a recursive manner. The process is completed when the subset at a node has all the same value of the target. The ensemble of 200 trees have been created, all trained on random set of observations. The actual input vector was adjusted to the majority class pointed by these trees.

6.3 Random forest. The random forest is an ensemble of many multivariate decision trees [23]. In distinction to DT the method combines "bagging" idea and the random selection of few variables (features) in each node to construct a collection of decision trees with controlled variation. In this way each tree in the forest is constructed in a way providing the highest degree of independence. The decision trees are trained on part of the available data and output the class that is the mode of the classes indicated by individual decision trees.

\section{Numerical results of experiments}

Selected diagnostic features representing sets depicted in Table 1 were applied to three mentioned above classifiers, responsible for recognition of classes (class 1 - sharp state of drill and class 2 - worn out drill). In numerical experiments the leaveone-out strategy was applied. In this strategy one observation is used for testing and the rest in the learning process of the classifier. After some introductory experiments with SVM the following values of the hyper-parameters $C=100$ and $\sigma=10$ of the Gaussian kernel have been adjusted. In DT and RF systems 200 trees have been used.

The data set taking part in experiments contained 102 observations regarding the sharp state of drill and 145 observations representing different degrees of blunting (worn out state). All of them have been mixed and taken part in learning and testing the classification systems by applying leave-one-out mode of operation.

The statistical results of numerical experiments concerning class recognition at application of different types of wavelet families (as presented in Table 1) are presented in Table 2. The

Table 2

Comparative results of class recognition in application of different classifiers for the best selected descriptors shown in Table 1

\begin{tabular}{|c|c|c|c|c|}
\hline No & $\begin{array}{c}\text { Applied } \\
\text { wavelet }\end{array}$ & $\begin{array}{c}\text { Best } \\
\text { level }\end{array}$ & $\begin{array}{c}\text { Accuracy [\%] } \\
\text { DT/SVM/RF }\end{array}$ & $\begin{array}{c}\text { Standard deviation [\%] } \\
\text { DT/SVM/RF }\end{array}$ \\
\hline 1 & $\mathrm{db} 1$ & 2 & $91.10 / 93.91 / 95.14$ & $4.16 / 3.24 / 1.10$ \\
\hline 2 & $\mathrm{db} 5$ & 2 & $91.48 / 94.73 / 95.12$ & $4.41 / 2.32 / 2.34$ \\
\hline 3 & $\mathrm{db} 20$ & 2 & $89.50 / 95.13 / 95.13$ & $7.75 / 4.46 / 3.08$ \\
\hline 4 & coif4 & 2 & $91.10 / 93.55 / 95.15$ & $4.85 / 3.53 / 1.78$ \\
\hline 5 & sym2 & 2 & $91.10 / 94.34 / 95.13$ & $4.59 / 3.83 / 3.05$ \\
\hline 6 & Meyer & 2 & $91.93 / 94.31 / 94.33$ & $6.63 / 6.01 / 4.61$ \\
\hline 7 & rbio1.3 & 2 & $91.90 / 93.12 / 95.96$ & $2.50 / 2.26 / 1.39$ \\
\hline
\end{tabular}


results are given for the testing data only in the form of mean accuracy and standard deviation. The accuracy and standard deviation are given in the following order DT/SVM/RF (first DT, then SVM and finally RF).

The best accuracy (95.96\%) of class recognition was obtained by RF at application of features corresponding to biorthogonal wavelet rbio1.3 in combination with time and frequency domain descriptors shown in the last row of Table 1. This set of features has also provided the smallest value of standard deviation $(1.39 \%)$ of classification results in all runs of algorithm.

These classification experiments have been performed for separate sets of features corresponding to application of different wavelet families. In the next phase we have combined all generated features together and performed once again the selection process. The aim of the selection was to choose the optimal set of features from all generated ones. Application of step-wise forward and backward selection procedure has resulted in the set of features depicted in Table 3.

Table 3

Set of feature subeset from all available potential diagnostic features

\begin{tabular}{|l|}
\hline \multicolumn{2}{|c|}{ Selected features } \\
\hline FFT5_AE, FFT5_M, FFT7_M, FFT2_F, FFT10_C, \\
Mean_F, StdF, VarAE, VarF, HistAE5, HistAE6, \\
HistAE10, HistM1, HistF, HistF7, HistF9, HistV5, \\
SkewnessC, KurtosisM, RMS_AE, EnergyM2, \\
EnergyF, EnergyM2, EnergyC2, EnergyC2, EnergyC3, \\
EnergyC3, EnergyM1
\end{tabular}

Application of this set of features has resulted in slightly different results, presented in Table 4. The accuracy of class recognition of the RF classifier has been increased to the value of $96.35 \%$. This is the best result of recognition achieved.

Table 4

Result of classification for selected features from all available sets

\begin{tabular}{|c|c|}
\hline $\begin{array}{c}\text { Accuracy [\%] } \\
\text { DT/SVM/RF }\end{array}$ & $\begin{array}{c}\text { Standard deviation [\%] } \\
\text { DT/SVM/RF }\end{array}$ \\
\hline $91.13 / 94.36 / 96.35$ & $5.87 / 4.32 / 3.00$ \\
\hline
\end{tabular}

\section{Conclusions}

In the paper an automatic system for assessing the state of the drill in a standard laminated chipboard drilling process was presented. We have shown that the satisfactory recognition of drill state is possible on-line by using sensor signals registering the feed force, cutting torque, noise, vibration and acoustic emission. Based on these signals the diagnostic features have been defined. The definition is based on time domain, Fourier and wavelet packet descriptions. This diversified approach to feature generation allows for describing the drill wear in a more effective way. The selection process applying the se- quential feature algorithm led to choosing the most significant features, which were used as the input attributes to the classification system.

Three different classifiers: SVM, DT and RF have been checked in the role of classifier. Based on many performed experiments, we have found that application of the RF classifier combined with the proper feature set provides the best recognition of the drill state (the highest accuracy rate). The relative testing error of recognition in leave-one-out mode of operation was below $4 \%$.

\section{REFERENCES}

[1] C. Scheffer, H. Kratz, P. S. Heyns and F. Klocke, "Development of a tool wear-monitoring system for hard turning", International Journal of Machine Tools \& Manufacture 43, 973-985 (2003).

[2] P.N. Botsaris and J.A. Tsanakas, "State-of-the-art in methods applied to tool condition monitoring (TCM) in unmanned machining operations: a review", Proceedings of the International Conference of COMADEM, Prague, 73-87 (2008).

[3] D. E. Dimla and P. M. Lister, "On-line metal cutting tool condition monitoring. I: force and vibration analyses", International Journal of Machine Tools \& Manufacture. 40, 739-768 (2000).

[4] N. H. Abu-Zahra and G. Yu, "Gradual wear monitoring of turning inserts using wavelet analysis of ultrasound waves", International Journal of Machine Tools \& Manufacture. 43(4), 33-343 (2003).

[5] K. Jemielniak, T. Urbański, J. Kossakowska J. and S. Bombiński, "Tool condition monitoring based on numerous signal features", Int J. Adv. Manuf. Technol. 59, 73-81 (2012).

[6] R. Lemaster, L. Lu and S. Jackson, "The use of process monitoring techniques on a CNC wood router. Part 1. Sensor selection", Forest Products Journal 50(7/8), 31-64 (2000).

[7] R. G. Silva, K. J. Baker and S. J. Wilcox, "The adaptability of a tool wear monitoring system under changing cutting conditions", Mechanical Systems and Signal Processing 14, 287-298 (2000).

[8] J. Wilkowski and J. Górski, "Vibro-acoustic signals as a source of information about tool wear during laminated chipboard milling", Wood Research 56(1), 57-66 (2011).

[9] R. J. Kuo, "Multi-sensor integration for on-line tool wear estimation through artificial neural networks and fuzzy neural network", Engineering Applications of Artificial Intelligence 13, 249-261 (2000).

[10] C. Scheffer and P. S. Heyns, "Wear monitoring in turning operations using vibration and strain measurements", Mechanical Systems and Signal Processing 15(6), 1185-1202 (2001).

[11] A. Noori-Khajavi and R. Komandur, "Frequency and time domain analyses of sensor signals in drilling-Part I", International Journal of Machine Tools and Manufacture 35(6), 775-793 (1995).

[12] N. H. Abu-Zahra and G. Yu, "Gradual wear monitoring of turning inserts using wavelet analysis of ultrasound waves", International Journal of Machine Tools \& Manufacture 43(4), 333-343 (2003).

[13] Q. Liu and Y Altintas, "On-line monitoring of flank wear in turning with multilayered feed-forward neural network", International Journal of Machine Tools \& Manufacture 39, 1945-1959 (1999). 
[14] S. S. Panda, A. K. Singh, D. Chakraborty and S. K. Pal, "Drill wear monitoring using back propagation neural network", Journal of Materials Processing Technology 172, 283-290 (2006).

[15] K. Patra, S. K. Pal and K. Bhattacharyya, "Artificial neural network based prediction of drill flank wear from motor current signals", Applied Soft Computing 7, 929-935 (2007).

[16] P. Lezanski, "An intelligent system for grinding wheel condition monitoring”, Journal of Materials Processing Technology 109, 258-263 (2001)

[17] J. H. Zhou, C. K. Pang, Z. W. Zhong, and F. L. Lewis, "Tool wear monitoring using acoustic emissions by dominant-feature identification", IEEE Transactions on Instrumentation and Measurement 60 (2), 547-559 (2011).
[18] M. V. Wickerhauser, Lectures on Wavelet Packet Algorithms, Washington University, 1991.

[19] I. Daubechies I., Ten Lectures on Wavelets, SIAM, Philadelphia, 1992.

[20] Matlab User Manual, Natick, MathWorks, 2014.

[21] V. Vapnik, Statistical Learning Theory, Wiley, New York, 1998.

[22] V. Kecman, Learning and Soft Computing: Support Vector Machines, Neural Networks, and Fuzzy Logic Models, MIT Press, Cambridge, 2001.

[23] T. Leś, S. Osowski, M. Kruk, "Automatic recognition of industrial tools using artificial intelligence approach", Expert Systems with Application 40, 4777-4784 (2013).

[24] L. Breiman, "Random forests", Machine Learning 45, 5-32 (2001). 